\title{
Integrated Structural Analysis Tool using the Linear Matching Method part 1 - Software Development
}

\author{
James Ure ${ }^{a}$, Haofeng Chen ${ }^{a, *}$, David Tipping ${ }^{b}$ \\ ${ }^{a}$ Dept of Mechanical and Aerospace Engineering, University of Strathclyde, 75 Montrose \\ Street, Glasgow G1 1XJ, Scotland, United Kingdom \\ ${ }^{b}$ Central Engineering Support, EDF Energy Nuclear Generation Ltd., Barnwood, Gloucester \\ GL4 3RS, United Kingdom
}

\begin{abstract}
A number of direct methods based upon the Linear Matching Method (LMM) framework have been developed to address structural integrity issues for components subjected to cyclic thermal and mechanical load conditions. This paper presents a new integrated structural analysis tool using the LMM framework for the assessment of load carrying capacity, shakedown limit, ratchet limit and steady state cyclic response of structures. First, the development of the LMM for the evaluation of design limits in plasticity is introduced. Second, preliminary considerations for the development of the LMM into a tool which can be used on a regular basis by engineers are discussed. After the re-structuring of the LMM subroutines for multiple central processing unit (CPU) solution, the LMM software tool for the assessment of design limits in plasticity is implemented by developing an Abaqus CAE plug-in with graphical user interfaces. Further demonstration of this new LMM analysis tool including practical application and verification is presented in an accompanying paper.

Keywords: Linear Matching Method (LMM), Structural Integrity, Load Carrying Capacity, Shakedown limit, Ratchet limit, Steady State Cycle

\footnotetext{
${ }^{*}$ Corresponding author. Tel.: +44 1415482036

E-mail address: Haofeng.chen@strath.ac.uk
} 


\section{Introduction}

Many Engineering components and structures in defence, aerospace, petrochemical, automobile and power industries operate under cyclic thermal and mechanical load conditions, and would include such diverse products as advanced internal combustion (IC) engine and gas turbine components, high altitude ramjet and rocket motors, chemical reactor vessels in plastics manufacture, prototype fusion reactors, power boilers, etc. In all of these applications, it is important to ensure the equipment can operate safely for the specified design life under the arduous environmental conditions. This requires engineers to identify possible failure mechanisms and guard against these during the design process [1]

Engineering structures subjected to cyclic loading histories will experience either elastic/plastic shakedown or ratchetting depending upon the applied load level. Limiting the behaviour of the structure or component to the elastic range is not an effective approach to a problem, as this leads to over-conservative design. In many applications, it is acceptable to allow limited plastic deformation to occur provided it can be shown that the structure shakes down to elastic action in the first few cycles of load. If the applied load is below the elastic shakedown limit, ratchetting and plastic shakedown will not occur under repeated loading. However in some situations, for example in nuclear power applications with cyclic thermal loading, this elastic shakedown limit can also be over-conservative. Thus an alternative approach is required to allow plastic shakedown to occur but preclude ratchetting. In ratchetting, a net increment of plastic strain occurs with each cycle of load and leads to an incremental plastic collapse over a number of cycles. Guarding against incremental plastic collapse by the determination of plastic shakedown limit or ratchet limit is crucial in any design involving cyclic thermal and mechanical loads. Under plastic shakedown condition, a low cycle fatigue (LCF) analysis would also be undertaken to ensure the structure does not fail by low cycle fatigue associated with local alternating plasticity, where the number of cycles to failure is determined by the maximum plastic strain range. A steady state cyclic analysis is often sought to evaluate the LCF life and address whether shakedown or ratchetting occurs under the defined cyclic load condition.

Design limits in plasticity for components subjected to cyclic thermal and mechanical load conditions, including both shakedown and ratchet limits, have attracted the attention of many researchers. The incremental finite element (FE) analysis [2] allows the investigation of any type of load cycle but inevitably requires significant computer effort for complex 3D structures. There has been a recent trend towards the development of direct methods that 
combine the convenience and efficiency of rule based methods [1] and the accuracy of incremental FE simulation techniques. Of these simplified direct methods [3-9], the Linear Matching Method (LMM) [10-14] has become one of the most powerful numerical methods for generating approximate inelastic solutions and answering specific design related issues using standard finite element codes. The basis of the LMM is through an idea of representing histories of stress and inelastic strain as the solution of a linear problem, where the linear moduli are allowed to vary both spatially and in time. The LMM has been formulated and implemented for the evaluation of shakedown limit $[10,11]$ and ratchet limit $[12-13]$. And more recently, a new LMM framework was developed to evaluate the steady state cyclic behaviour of component for the LCF assessment purpose [14].

The LMM is distinguished from the other upper bound or lower bound direct methods by ensuring that both the equilibrium and compatibility conditions are satisfied to produce exact solution at each stage of calculations [13], and is counted to be one of the methods most amenable to practical engineering applications involving complicated thermomechanical load history [15]. However, as many other direct methods, the LMM was initially implemented into commercial FE software Abaqus [2] using user subroutines developed by FORTRAN programming language, which is difficult for non-experts to operate. Another drawback of this implementation is the level of programming experience required to create and submit an analysis: the alterations to the subroutines required to run each analysis present issues for everyday use by engineers generally not familiar with FORTRAN. To remedy this situation and enable widespread adoption of the LMMs in industry, an integrated software tool is required to not only removes the requirement for manual subroutine alterations, but also provide additional functionality for subsequent life assessment calculations.

The main objective of this paper is to develop a new integrated structural analysis tool using the LMM framework for the assessment of design limits in plasticity, including the evaluation of limit load, shakedown limit, ratchet limit and steady state cyclic behaviour of the structure. It will deliver the LMM in a form where it can be readily used by engineers with the responsibility for design and life assessment decisions on a regular basis. The software tool would allow engineers to access the LMM solution methods without having to make any of the changes to the subroutines required to run a LMM analysis as was previously required. 
The paper is organized as follows. In Section 2, preliminary considerations are discussed by examining previous LMM versions and the way of customising Abaqus. In Section 3, a restructuring of the LMM user-subroutines for multiple CPU solution is proposed. The creation of a Graphical User Interface (GUI) via an Abaqus Plug-in is presented in Section 4. Section 5 briefly discusses the installation and testing of the LMM software tool for the assessment of design limits in plasticity. Section 6 concludes this paper.

\section{Preliminary Considerations}

\subsection{Previous LMM Versions}

The original incarnation of the LMM code was created as Abaqus user subroutines and has been mainly used for research purposes. A typical LMM analysis consists of two stages [1213]. In the first stage an elastic analysis for each applied load and temperature distribution is performed using the elastic analysis UMAT subroutine. For each of these analyses the elastic stress tensor for each integration point is written to a text file, and the integration point temperature is written to a separate text file. The second stage of this analysis uses a second UMAT subroutine and these text files to perform the shakedown or ratchet limit calculation. In this second stage some changes are required to the UMAT code in order to set up the analysis. For example, the number of integration points per element and the total number of elements in the model needed to be changed so that the arrays could be sized appropriately. The code defining the load cycle also requires updating, which reads the stress and temperature text files to generate the applied stresses at each point in the load cycle.

For an analysis using this set of subroutines both the elastic analyses and the further shakedown or ratchet calculation are submitted using the Abaqus batch command i.e. the Abaqus input file for the model is required. This input file is generated using Abaqus CAE for a complete model. The majority of the content of the input file is common between the elastic and shakedown calculations (such as geometry and boundary conditions). However there are some differences which must be performed manually (such as requesting the energy outputs associated with a UMAT subroutine). A further upgrade of these subroutines was carried out [16] so that the LMM could be used with minimal code changes, where the load cycle is defined via a formatted text file which was read by the subroutines. This significantly reduces the code changes required for an analysis. However, the changes to the Abaqus input file still needed to be performed manually.

The creation of a formatted text file to configure the LMM analysis was a major step in the usability of the LMM, and in fact draws a parallel with the way in which any conventional 
Abaqus analysis operates. In an Abaqus analysis, a FE model needs to be created in Abaqus CAE and submitted for analysis, although other pre and post processors are often used. Abaqus CAE then creates a formatted text file (Abaqus input file) which is passed to the Abaqus solver for solution. The text file for the LMM analysis is equivalent to the input file, the only difference being that it is created manually. The creation of a text file is also adopted here as it is a simple and robust method for passing information from the LMM user interface into the subroutines. The major aim of this software tool is that the text file is generated by the user interface rather than manually.

\subsection{Customising Abaqus}

Abaqus [2] contains a large number of options for the user to customise a model or analysis for their particular situation. To obtain user-generated solution options the user-subroutines can be used, which is how the LMM has been implemented. In addition to this Abaqus CAE contains the option to use scripts to perform operations on the model or results databases. These scripts are written in the Python open source scripting language [2], and Abaqus has extended this language to allow operations to be performed within CAE itself. These scripts can be used to perform all operations which are available through the CAE interface (i.e. applying loads, meshing, plotting results etc.) and can also query the model or Abaqus output file (odb) for values. A typical example where scripts serve a useful function is in a sensitivity analysis, where an automatic process can vary a particular value in a model, resubmit for solution, query the results and decide whether a further iteration is required.

The use of python scripting within Abaqus is a very powerful tool, because options also exist to use this language to customise the CAE user interface itself. This can be achieved by creating either an entirely custom CAE interface or a plug-in to the standard CAE. The ability to create a custom GUI is a powerful tool as the modules and toolsets which are not desired can be removed and custom functions can be added. Abaqus Viewer is an example of this, where all the analysis toolsets and modules have been removed, leaving only the visualisation module for viewing output databases. Plug-ins form another useful avenue of adding functionality to the Abaqus CAE interface. Plug-ins can be created for a variety of purposes; commonly they are used to streamline tasks which are complex to perform or are performed regularly. For the LMM application a plug-in has been selected over a custom CAE interface. A plug-in can be accessed through the conventional CAE interface during normal use, whereas a custom CAE would need to be opened separately. An engineer with an 
existing Abaqus model will be able to access the LMM through $C A E$, and the plug in will guide them through the process of entering the information needed to run a LMM analysis.

The creation of a plug-in to gather the required data and format the model will require a set of subroutines which are compatible with this model configuration and the text file used to pass in the data for the analysis. The re-structuring of the subroutines to accommodate this presents an opportunity to re-write them for multiple CPU solution, especially since even basic desktop computers now have dual or quad core as standard. Some features of the UMAT code written by Chen [12-14] and Tipping [16] are not amenable to solution with multiple CPUs.

With all of these considerations, a plug-in has been created and the LMM subroutines have been re-written. The plug-in posts dialog boxes to gather the required information and data from the user. When the process is complete, the plug-in configures the model for the LMM analysis using scripts. The plug-in also writes the text file containing relevant data for the subroutines. The subroutines themselves have been re-written to allow multiple CPU solution in the UMAT routine.

The structure and function of the plug-in is dictated by many of the features of the subroutines and their re-structuring for multiple CPU solution. Therefore the subroutines will be described in the next section, followed by a description of the plug-in created to use them.

\section{Re-structuring of the Subroutines}

Figure 1 shows the general structure of a LMM solution with the new set of subroutines for various plastic analyses. The starting point of this process assumes that the plug-in has created a formatted text file containing information about the analysis such as the convergence tolerance, analysis type, load cycle and temperature dependent material data. This LMM text file produced by the plug-in will then be read by the subroutine at the beginning of the analysis. This means that the UMAT subroutine is called for each integration point which has been defined as a User-material from within Abaqus CAE. Defining areas of the model as a User-material within Abaqus CAE tells the solver to look to the UMAT subroutine for the material behaviour of those areas. This solution stage of the analysis uses the number of CPUs requested by the user, and so this UMAT subroutine must be coded to accommodate this. 
As part of the restructuring, all the elastic calculations for the applied loads and the LMM calculation itself have been incorporated into a single Abaqus analysis. Each elastic calculation is carried out as a single analysis step within this Abaqus analysis, with the LMM calculation being performed in the final analysis step. This consolidation into a single Abaqus analysis means that the passing of stresses and temperatures in text files is no longer required. Instead the results file itself can be used for storage and access of this information. When defining a User-material in Abaqus CAE, the user is able to specify the number of Solution-Dependent State Variables (SDV) for that material. This is the number of memory spaces available to the UMAT in the output database file, and so is commonly used to provide contour plots of user defined variable fields calculated during the UMAT solution. However, the direct access of SDVs within UMAT means that they can also be used as a way of storing values and data to be used during the analysis. Hence this has been used to pass elastic stresses and temperatures between the elastic analyses and the LMM analysis, removing the need for text files and therefore removing this restriction to multiple CPU solution.

Returning to Figure 1, the UMAT subroutine is divided into elastic and LMM sections. An elastic analysis is performed for each applied load and the stresses and temperatures are stored in the SDV slots. When all applied loads have been considered, an intermediate "null" step is included. This allows key variables in the subroutine to return to zero before commencing the LMM solution. The LMM solution itself uses the stresses and temperatures from the SDVs along with the load cycle and material property data read in the UEXTERNALDB subroutine to perform the calculations.

Within the LMM solution stage, the data previously stored in model-sized arrays is now stored using the SDVs. Abaqus itself manages the multiple CPUs accessing the results files as it would during any analysis which uses multiple CPUs. These existing methods for managing multiple CPU solution mean that the UMAT subroutine can use Abaqus itself to manage the multiple CPUs accessing the data simultaneously. The alternative, placing these model-sized arrays into common memory, would mean the CPUs would have to queue for access to the array.

The URDFIL subroutine is called by Abaqus at the end of every increment, and plays a number of key roles. Firstly, the URDFIL can be used to access the results file and so is able to obtain the volume integrals required to calculate the upper bound multipliers of equations (16) in [11] and (29) in [12]. Being called at the end of the increment means that 
the URDFIL is used to provide a summary of the increment to the user to give an indication of how the solution is progressing. Finally, the URDFIL routine can also be used to terminate an analysis. Convergence calculations are performed in URDFIL and if the convergence criteria are satisfied then the analysis is ended. If convergence is not met, then the solution continues for a further increment where the UMAT is called and the LMM calculations are performed once again.

With these subroutines there are three LMM analyses possible for the design limits in plasticity: strict shakedown, steady state cycle only and ratchet limit (i.e. the global shakedown limit). It is worth noting that when having only one load time point, the shakedown analysis automatically reduces to a limit analysis, and the limit load or load carrying capacity of the component can be evaluated as a special case of this shakedown analysis. The subroutines have been programmed to be flexible and allow as many options as possible within these three analyses. These options are summarised in Table 1. As shown in Table 1, the calculation of the ratchet limit includes two stages of calculation [12], the first a steady state cyclic analysis for a cyclic history of residual stress and the second for the ratchet limit due to an extra constant load. This set of subroutines require that the Abaqus model is configured in a certain way (e.g. one analysis step per applied load) and so provides a set of requirements which has dictated the design of the plug-in and its operation.

\section{Graphical User Interface via an Abaqus Plug-in}

Upon selecting the LMM plug-in from the "plug-ins" menu in Abaqus CAE (Figure 2), the user is then guided through the Main dialog box seen in Figure 3. The Main dialog box is posted which prompts the user to select which model from within the current CAE session they would like to analyse and which type of LMM analysis - strict shakedown, steady state cycle or steady state cycle + ratchet limit. Selecting a steady state cycle analysis means that only stage 1 of the ratchet analysis procedure [12] is performed to give the steady cyclic state of the component along with the associated strain ranges, which provide information concerning fatigue crack initiation in low cycle fatigue. The second stage, to find the ratchet limit is not performed when selecting a steady state cycle analysis.

With the information from this dialog, background scripts perform a series of checks on the selected model to ensure it is possible to perform a LMM analysis successfully. This includes very basic checks, such as ensuring the component is meshed. It also includes checks more specific to the LMM, such as ensuring that at least one mechanical load is applied when a 
ratchet limit analysis is selected. Any error found is displayed to the user so that it may be remedied as shown in Figure 3.

Upon passing these checks, a series of subsequent dialogs are posted. This begins with the material data. A Material dialog box is posted for each material which is used in the current model. Within each of these dialogs the user is prompted to enter the Young's modulus, yield stress, Poisson's ratio and the thermal expansion coefficient. The Young's modulus and yield stress may be temperature dependent, which is enabled by selecting this in a check box. In many situations the model will have already been used in a previous analysis, meaning material property data has already been defined in CAE. If this is the case the "Extract" function can be used, which queries the current material for the four properties required for the LMM analysis and populates the dialog box accordingly. The Material dialog box is shown in Figure 4, alongside some of the possible error messages.

When either a steady state cycle analysis or a ratchet analysis is chosen the user has an option of using a Ramberg-Osgood (R-O) plastic model. In this case the Material dialog is shown in Figure 5a. The Ramberg-Osgood parameters may be temperature dependent or independent in the same way as the modulus and yield stress. A function is also included to link the $\mathrm{R}-\mathrm{O}$ parameters to the yield stress. This function calculates the $0.2 \%$ proof stress from the R-O parameters entered and populates the yield stress fields accordingly. Additional advice is available for the R-O model by selecting the "Tip" button, which displays the box shown in Figure 5b. It is worth noting that each active material in the FE model will allow users to define the material properties through a separate Material dialog box, meaning that the LMM software tool is capable of analysing components or structures with multiple materials such as composites or welded components. When this is complete, the plug-in moves on from materials to the load cycle.

The definition of the load cycle is crucial for all analyses. This is done through the Load cycle dialog boxes, where the Loadcycle table at the top of the box is present. This table allows the user to define a load cycle by adding any number of time points and scaling the loads to the appropriate level for that time point in the cycle. At each time point a temperature field can also be applied by selecting desired predefined field from the drop-down list. Selecting the "Tip" button displays a box with additional information on populating this table and a simple worked example as shown in Figure 6. 
A subtle difference between the three analysis options comes when selecting the loads which may be scaled. In a strict shakedown analysis, any of the applied loads and temperature may be selected for scaling. These loads and temperature fields will be scaled by the load multiplier, $\lambda$, to find the strict shakedown limit. The loads which are not checked as scalable are left at the magnitudes given in the load cycle table. A steady state cycle analysis has no option for scaling loads. This is because the load levels given in the table are fixed at those values and a steady state cycle analysis determines the steady state response due to this predefined cyclic load condition. The ratchet analysis procedure requires that an additional steady state load is scaled to find the global shakedown limit. The user may select which of these loads are to be added as additional loads. Detailed advice on the load scaling for each analysis type is given in the "Tip" box (Figure 6).

The Analysis Parameters dialog shown in Figure 7 is the final dialog box and gathers the data required to create the analysis (such as the job name and working directory) and the solution controls (e.g. convergence level and maximum number of increments). The current working directory is automatically detected and displayed, but the user may choose to change this. Default values of convergence levels are also displayed for inexperienced users.

There are different convergence options available depending on the analysis type chosen. A strict shakedown analysis can use either the difference between consecutive upper bounds (which historically was the way convergence was judged) or can use the \% difference between lower and upper bounds. A steady state cycle convergence is based on volume integrals of modulus between consecutive increments. If there is little change in this value then the stress fields are not changing and so have reached a converged steady state behaviour. The value of convergence in the dialog box represents the percentage change of this volume integral in consecutive increments. A ratchet limit analysis requires convergence values for both stages of the procedure. Stage 1 is identical to that of a steady state cycle analysis, and the convergence options for stage 2 are identical to those for the strict shakedown analysis. Once again, the "Tip" box gives additional information and help to the user if required.

If the data entered in the Analysis Parameters box passes the error checking stage, then the user interface portion of the plug-in is complete. In total the dialog boxes will have gathered the following information: model name, analysis type, material properties (temperature dependent), load cycle, which loads are scalable, job name, max number of increments, convergence criteria and working directory. The next stage of the plug-in is then to use this 
information to configure the Abaqus model and write the LMM text file containing the information for the subroutines. The changes to the model have been designed so that the original model is entirely recoverable i.e. nothing of the original model is deleted. Instead items are suppressed or copied so that the LMM configuration of the model can be applied. All the model data is passed into a set of scripts which write relevant information to the LMM text file and perform a series of changes to the model.

Firstly, a LMM text file is created and populated with information about the analysis. An example of this is shown in Table 2. Once this information has been written to the LMM text file, the next stage is to configure the loads and boundary conditions in the Abaqus CAE model. Two basic requirements dictate the way in which this is achieved. The first requirement is that the elastic stress for each applied load must be known so that superposition can be used to construct the load cycle in the subroutines. The second requirement is that the same boundary conditions are used for all elastic analyses and the shakedown/steady cycle/ratchet analysis. Therefore one analysis step is created for each of these applied loads and predefined fields and the corresponding load or field is applied in isolation in that step. Figure 8 shows example Load Manager and Predefined Field Manager tables from Abaqus to illustrate this. A "Null" analysis step is created where no loads are applied, which allows the subroutines to return key variables to zero. The final analysis step created is for the shakedown/steady cycle/global shakedown analysis. This situation allows the LMM subroutines to obtain the elastic stresses for each load in turn, return the subroutine variables to zero and then carry out the shakedown/steady cycle/ratchet analysis. The boundary conditions are moved to the first step and set to propagate through the entire analysis. The original analysis steps are suppressed, allowing the user to recover the original state of the model.

The materials are the next area to receive attention. The LMM requires a User-material to be defined in Abaqus to link with the UMAT subroutine. In Abaqus CAE a copy of each active material is made which is defined as a User-material for the LMM analysis. The original material is retained so that the user may recover the original material properties. Values for temperature independent Young's modulus, Poisson's ratio, yield stress and the RambergOsgood parameters are entered as constants in this material. A User-material is only able to include multiple constants, not tables. Therefore if a property is temperature dependent then a value of zero is entered for this constant and the temperature dependent values are appended to the bottom of the LMM text file. An example of this is given in Table 3. 
After a materials configuration, the field and output requests are created. Once again any original requests in the model are suppressed rather than deleted. The most important of the LMM output requests is the variable SDV, which is the user-defined output from the UMAT subroutine. Requesting this output allows the user to view contour plots of the variables calculated within the UMAT routine, and so it is vital that this is included.

In addition to the field and history outputs, an energy file output must be requested so that the volume integrals needed for the upper bound load multiplier can be accessed. The only way to achieve this is by adding commands to the keyword block for the model, which contains all the commands printed to the input file when the analysis begins (shown in Figure 9). Part of this script inserts the commands to the keyword block as if a user had manually typed them. To recover the initial state of the model a user simply clicks the button "Discard All Edits", which removes any user-added commands.

The final script creates the LMM analysis job with the correct subroutine for the analysis type selected. When created, the user may edit the job in the same way as any other Abaqus job by selecting the number of CPUs to solve with, queue options etc. Finally the user may submit the analysis for solution. Whilst solving any Abaqus job the progress of the solution may be seen in the "Monitor" dialog box. Information such as the current step and increment are displayed along with any warnings and errors encountered. The URDFIL subroutine contains code which prints additional information about the LMM to the Data file which can be read by this dialog box. During the solution, the lower and upper bound load multipliers and convergence levels are printed for the current increment so the user can see the progress of the solution. When an analysis is complete a summary is printed which declares the shakedown status of the model, the final values of the load multipliers and lists the SDV numbering so the user can view contour plots of the results. A sample summary given in the Monitor dialog box is shown in Figure 10. When complete, the user may view contour plots of the results in the same way as any other Abaqus analysis.

\section{Installation and Testing}

The plug-in and subroutines described here have been installed and tested on the computer system of our industrial partner. Once it is successfully installed, this new integrated LMM structural analysis tool will take into effect in the Abaqus CAE as a plug-in shown in Figure 2. The independent users from industry have contributed to extensively test the LMM software tool and give feedback on any problems encountered or additional functions which would be of benefit. 
This external perspective was a valuable asset. A lot of effort was invested in error checking of the inputs of each dialog box. However it is very difficult for a single programmer to foresee every eventuality or combination of events which could lead to an error. The testing within the industrial partners highlighted some additional situations which should be avoided and minor errors with the plug-in itself, and these were rectified on site when they arose.

This testing also highlighted some small additional functions which would be beneficial in regular use. For example in Figure 5 a the function which calculates the $0.2 \%$ proof stress was added in this way. Therefore the plug-in overall has been tailored based on the requests from industry, making the tool more convenient for their engineers to use.

\section{Conclusions}

This paper has described the development of the LMM into an integrated structural analysis tool which can be used on a regular basis by industrial engineers for assessing the load carrying capacity, shakedown limit, ratchet limit and steady state cyclic behaviour of components subjected to cyclic thermal and mechanical load condition. This has involved restructuring the subroutines for multiple CPU solution and developing a plug-in for Abaqus CAE.

This plug-in tool has been created to provide an intuitive and simple way to perform a LMM analysis. The data for the analysis is gathered through Abaqus CAE, which is a familiar environment for industrial engineers. The plug-in implements all of the functionality possible in the subroutines, and includes extensive error checking to ensure that only permissible combinations of options are used. The plug-in and subroutines have been written so that the configuration of the subroutines for each analysis is performed automatically, thus making a LMM analysis more convenient and less prone to errors.

Further demonstration of this new LMM analysis tool including practical application and verification is presented in an accompanying paper [17]. 


\section{Acknowledgments}

The authors gratefully acknowledge the support of the Nuclear EngD Centre of the United Kingdom, EDF Energy and the University of Strathclyde during the course of this work. The authors would also like to thank Prof Alan Ponter from University of Leicester for his advice and discussions on the theoretical and software developments of the LMM.

\section{References}

[1] R5: An assessment procedure for the high temperature response of structures, Revision 3. British Energy Generation Limited, Gloucester, UK, 2003.

[2] Abaqus User's Manual. Dassault Systèmes Simulia Corp. 2009.

[3] Vu DK, Yan AM, Nguyen-Dang H. A primal-dual algorithm for shakedown analysis of structures. Computer Methods in Applied Mechanics and Engineering 2004; 193: 4663-4674.

[4] Muscat M, Mackenzie D, Hamilton R. Evaluating shakedown under proportional loading by non-linear static analysis. Computers \& Structures 2003; 81: 1727-1737.

[5] Ponter ARS, Carter KF. Shakedown state simulation techniques based on linear elastic solutions. Computer Methods in Applied Mechanics and Engineering 1997; 140: 259279.

[6] Adibi-Asl R, Reinhardt W. Non-cyclic shakedown/ratcheting boundary determination Part 1: Analytical approach. International Journal of Pressure Vessels and Piping 2011; 88: 311-320.

[7] Staat M, Heitzer M. LISA a European Project for FEM-based Limit and Shakedown Analysis. Nuclear Engineering and Design 2001; 206: 151-166.

[8] Spiliopoulos KV, Panagiotou KD. A direct method to predict cyclic steady states of elastoplastic structures. Computational Methods in Applied Mechanics and Engineering 2012; 223-224: 186-198.

[9] Maier G, Pastor J, Ponter ARS, Weichert D. Direct Methods of Limit and Shakedown Analysis. Comprehensive Structural Integrity Volume 3, de Borst R, Mang HA. (Eds) Amsterdam: Elsevier Pergamon, 2003; 637-684. 
[10] Chen HF, Ponter ARS. Shakedown and limit analyses for 3-D structures using the linear matching method. International Journal of Pressure Vessels and Piping 2001; 78: 443451.

[11] Chen HF. Lower and Upper Bound Shakedown Analysis of Structures with Temperature Dependent Material Properties. ASME Journal of Pressure Vessel Technology 2010; 132(1): 011202.

[12] Chen HF, Ponter ARS. A Direct Method on the Evaluation of Ratchet Limit. ASME Joutnal of Pressure Vessel Technology 2010; 132(4): 041202.

[13] Chen HF, Ure J, Tipping D. Calculation of a Lower Bound Ratchet Limit Part 1 - Theory, Numerical Implementation and Verification. European Journal of Mechanics - A/Solids 2013; 37: 361-368.

[14] Chen HF, Ponter ARS. Linear matching method on the evaluation of plastic and creep behaviours for bodies subjected to cyclic thermal and mechanical loading. International Journal for Numerical Methods in Engineering 2006; 68(1): 13-32.

[15] Chen HF, Ponter ARS. Structural integrity assessment of superheater outlet penetration tubeplate. International Journal of Pressure Vessels and Piping 2009; 86(7): 412-419.

[16] Tipping D. The Linear Matching Method: A Guide to the ABAQUS User Subroutines. British Energy Generation Ltd Report E/REP/BBGB/0017/GEN/07, 2008.

[17] Chen HF, Ure J. Integrated Structural Analysis Tool using the Linear Matching Method part 2 - Application and Verification. International Journal of Pressure Vessels and Piping 2014. 
Table 1 Functions available in each LMM analysis

\begin{tabular}{|l|c|c|c|}
\hline \multicolumn{1}{|c|}{ Analysis Option } & $\begin{array}{c}\text { Strict } \\
\text { Shakedown }\end{array}$ & $\begin{array}{c}\text { Steady } \\
\text { State Cycle } \\
\text { Only }\end{array}$ & $\begin{array}{c}\text { Steady State } \\
\text { Cycle + } \\
\text { Ratchet Limit }\end{array}$ \\
\hline $\begin{array}{l}\text { All structural continuum element types (3D, } \\
\text { axisymmetric, plane strain and plane stress) }\end{array}$ & $\checkmark$ & $\checkmark$ & $\checkmark$ \\
\hline $\begin{array}{l}\text { Temperature dependent Young's modulus } \\
\text { and yield stress }\end{array}$ & $\checkmark$ & $\checkmark$ & $\checkmark$ \\
\hline Perfect Plasticity Material Model & $\checkmark$ & $\checkmark$ & $\checkmark$ \\
\hline $\begin{array}{l}\text { Ramberg-Osgood Material Model (which } \\
\text { may also be temperature dependent) }\end{array}$ & $\checkmark$ & $\checkmark$ & $\checkmark$ \\
\hline $\begin{array}{l}\text { Any number of points possible in the load } \\
\text { cycle }\end{array}$ & $\checkmark$ & & $\checkmark$ \\
\hline $\begin{array}{l}\text { Ability to select which loads to scale during } \\
\text { solution }\end{array}$ & $\checkmark$ & $\checkmark$ & $\checkmark$ \\
\hline Two convergence options (see section 4) & $\checkmark$ & & $\checkmark$ \\
\hline
\end{tabular}


Table 2 LMM text file example

\begin{tabular}{|c|c|}
\hline LMM Text File & Explanation \\
\hline $\begin{array}{l}\text { Analysis Type: } \\
\text { STRICT_SHAKEDOWN }\end{array}$ & $\begin{array}{l}\text { The analysis type. May also have a value of } \\
\text { STEADY_CYCLE_ONLY or } \\
\text { STEADY_CYCLE_AND_RATCHET }\end{array}$ \\
\hline $\begin{array}{l}\text { Max Number of Increments: } \\
350\end{array}$ & $\begin{array}{l}\text { Max number of increments defined by the user } \\
\text { should convergence prove elusive }\end{array}$ \\
\hline $\begin{array}{l}\text { Convergence (\%Diff) } \\
5.00000\end{array}$ & $\begin{array}{l}\text { The convergence option chosen and the value. The } \\
\text { (\%Diff) flag is present when the percentage } \\
\text { difference convergence option is used. It is not } \\
\text { present when the difference between upper } \\
\text { bounds is selected }\end{array}$ \\
\hline $\begin{array}{l}\text { Dimensionality } \\
\text { THREE_D }\end{array}$ & $\begin{array}{l}\text { Dimensionality flag. May also have a value of } \\
\text { THREE_D, AXISYMMETRIC, } \\
\text { PLANE_STRESS or PLANE_STRAIN. }\end{array}$ \\
\hline $\begin{array}{l}\text { Number of Applied Loads } \\
4 \\
\text { Number of Load Instances } \\
2\end{array}$ & $\begin{array}{l}\text { The number of loads (including temperature } \\
\text { distributions) and load instances in the cycle }\end{array}$ \\
\hline $\begin{array}{l}\text { Load: Bending Moment } \\
\text { NOT_SCALABLE } \\
0.00000 \\
1.00000 \\
\text { Load:Interna1 Pressure } \\
\text { SCALABLE } \\
0.00000 \\
1.00000 \\
\text { Load:Tension } \\
\text { SCALABLE } \\
0.00000 \\
0.50000 \\
\text { LOad:Temperature Difference } \\
\text { NOT_SCALABLE } \\
0.00000 \\
1.00000\end{array}$ & $\begin{array}{l}\text { The multipliers for each load as entered in the } \\
\text { Loadcycle table. The LMM uses these multipliers } \\
\text { to construct the load cycle in the code and so } \\
\text { determine the elastic stress at each load point. } \\
\text { The SCALABLE/NOT_SCALABLE flag dictates } \\
\text { whether the load can be scaled using the } \\
\text { calculated } \lambda \text { in each increment. }\end{array}$ \\
\hline
\end{tabular}


Table 3 Material properties section of the LMM text file

\begin{tabular}{|c|c|}
\hline LMM Text File & Explanation \\
\hline $\begin{array}{l}\text { Material Properties: } \\
\text { Number of materials: } 3\end{array}$ & $\begin{array}{l}\text { The number of materials is printed } \\
\text { so that UEXTERNALDB knows how } \\
\text { many materials to look for in the file }\end{array}$ \\
\hline $\begin{array}{l}\text { Material:SA508 } \\
\text { Temperature Independent Modulus } \\
\text { Temperature Independent Yield Stress } \\
\text { Perfectly Plastic Material }\end{array}$ & $\begin{array}{l}\text { The material name is printed to be } \\
\text { read by UEXTERNALDB. } \\
\text { The modulus and yield for each } \\
\text { material may be either: } \\
\text { Temperature Independent or } \\
\text { Temperature Dependent. } \\
\text { Temp independent values are } \\
\text { written to the User Material in } \\
\text { Abaqus }\end{array}$ \\
\hline 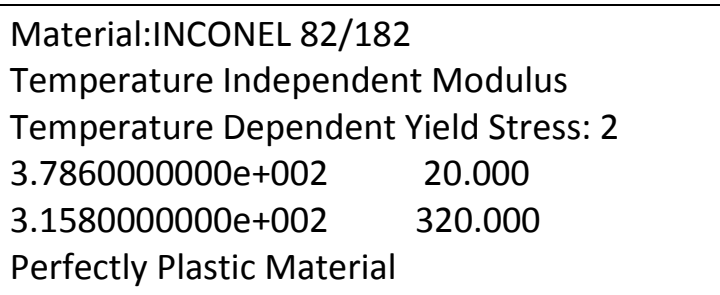 & $\begin{array}{l}\text { Where temperature dependent } \\
\text { properties are found, these are } \\
\text { printed in a formatted way so that } \\
\text { UEXTERNALDB is able to read them. }\end{array}$ \\
\hline $\begin{array}{l}\text { Material:STEEL } 316 \\
\text { Temperature Independent Modulus } \\
\text { Temperature Independent Yield Stress } \\
\text { Temperature Independent Ramberg-Osgood }\end{array}$ & $\begin{array}{l}\text { For each material, the words } \\
\text { Perfectly Plastic Material are printed } \\
\text { unless a Ramberg-Osgood material } \\
\text { is selected. } \\
\text { Where a Ramberg-Osgood model is } \\
\text { used with temperature dependent } \\
\text { properties then a formatted list of } \\
\text { these is provided in the same way as } \\
\text { modulus or yield. Otherwise it is } \\
\text { declared as temperature } \\
\text { independent and the values are } \\
\text { written in the User Material. }\end{array}$ \\
\hline
\end{tabular}




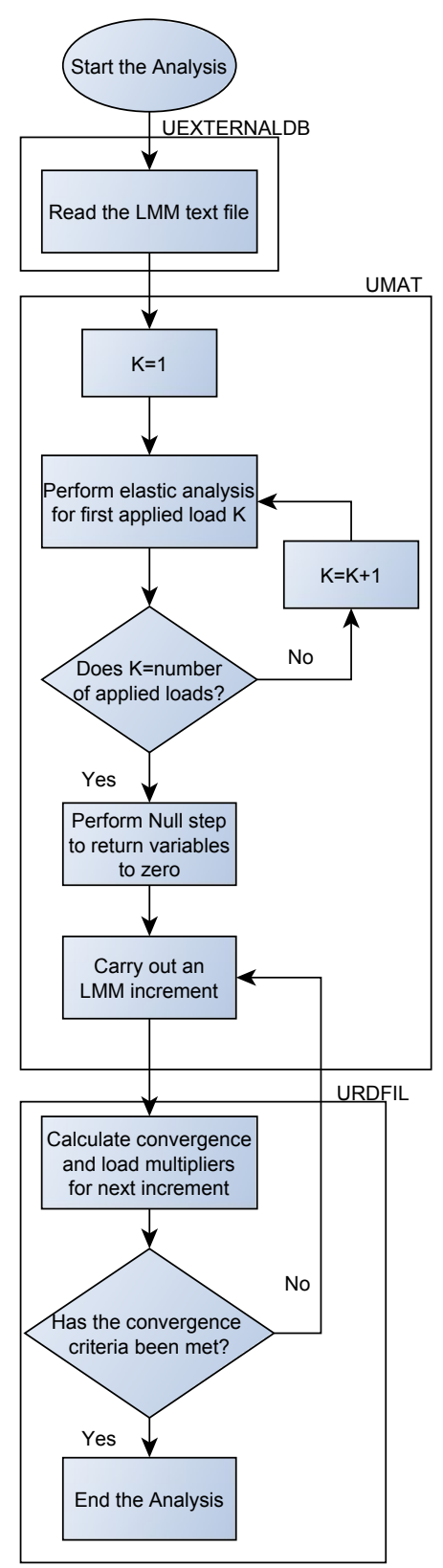

Fig. 1. Overall structure of a LMM solution 


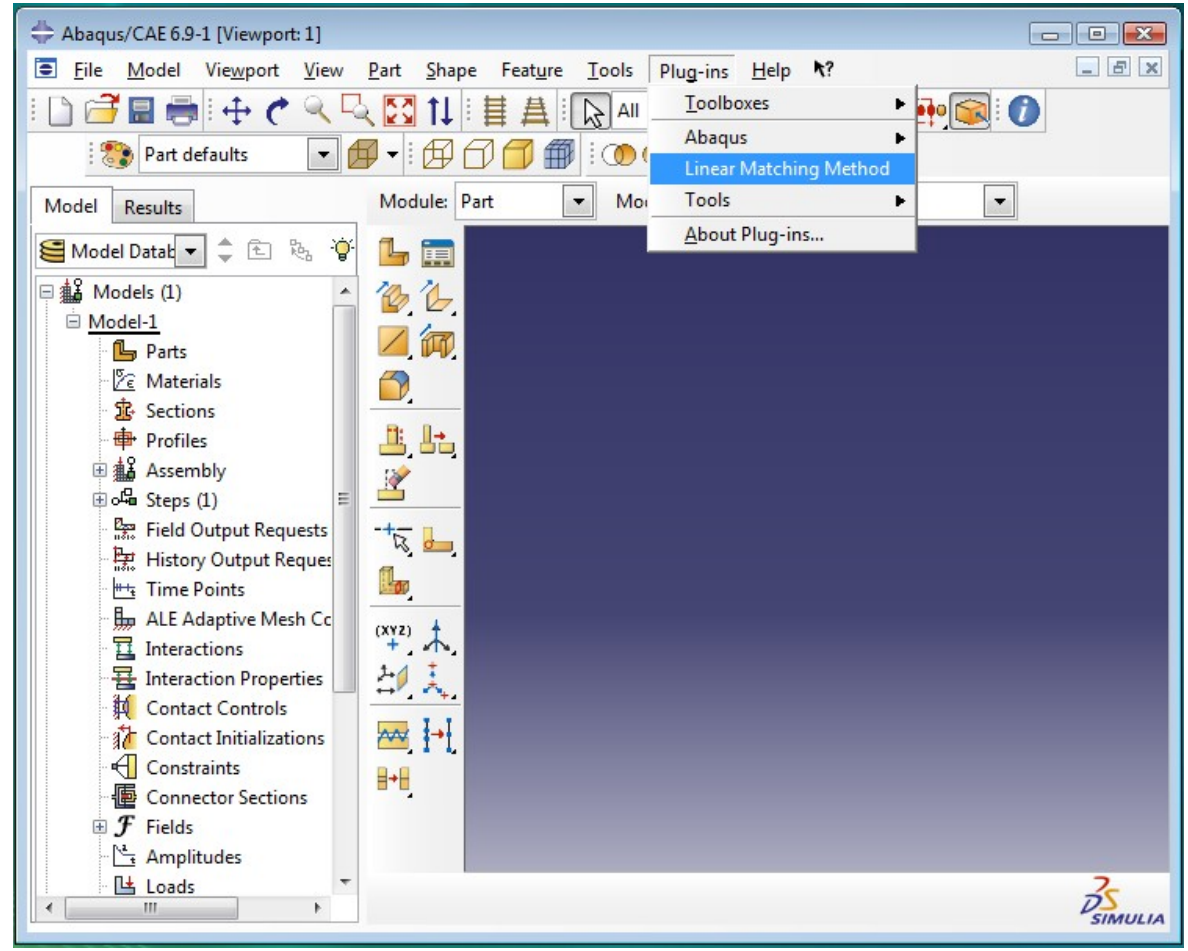

Fig. 2. An Abaqus CAE interface showing the linear matching method plug-in 


\begin{tabular}{|c|c|c|}
\hline \multicolumn{3}{|c|}{ II. Linear Matching Method } \\
\hline \multicolumn{3}{|c|}{ The Linear Matching Method } \\
\hline \multicolumn{3}{|c|}{$\begin{array}{l}\text { The Linear Matching Method (LMM) is an analysis technique which determines the } \\
\text { steady state response of structures subject to cyclic loading to find the strict } \\
\text { shakedown and ratchet limits. }\end{array}$} \\
\hline \multicolumn{3}{|c|}{$\begin{array}{l}\text { This plug-in takes material and load cycle data and then automatically configures } \\
\text { the Abaqus CAE model for an analysis with the LMM user subroutines. }\end{array}$} \\
\hline \multicolumn{3}{|c|}{ To begin the LMM process, please select a model and analysis type below: } \\
\hline Select Model & & Select Analysis Type \\
\hline \multirow{2}{*}{\multicolumn{2}{|c|}{$\begin{array}{l}\text { Please select a model to analyse with } \\
\text { the Linear Matching Method: }\end{array}$}} & (-) Strict Shakedown \\
\hline & & Steady State Cycle \\
\hline Select Model: & $\rightarrow$ & Steady State Cycle + Ratchet Limit \\
\hline \multicolumn{3}{|c|}{$\begin{array}{l}\text { The LMM subroutines and plug-in have been created by Haofeng Chen and James Ure. Any enquiries } \\
\text { should be directed to haofeng. chene brath. ac.uk. Use of this method in at your own risk. Both the } \\
\text { developers and the University of Strathclyde take no responsibility for any loss incurred directly or } \\
\text { indirectly through use of the LMM. }\end{array}$} \\
\hline \multicolumn{3}{|c|}{ (C) Copyright 2013 LMM. All Rights Reserved (www.thelmm.co.uk) } \\
\hline \multicolumn{3}{|c|}{ Continue... } \\
\hline
\end{tabular}
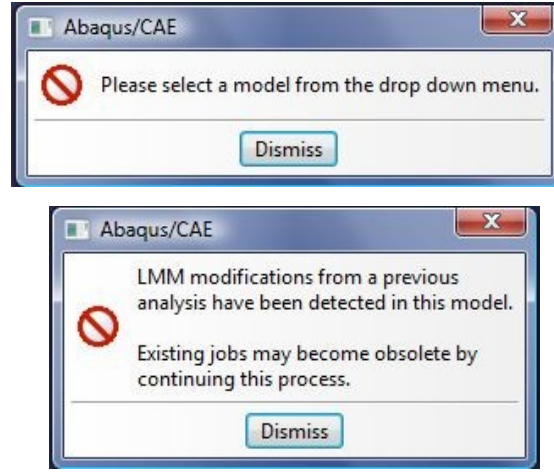

\begin{tabular}{|c|} 
At Abaqus/CAE \\
$\begin{array}{l}\text { At lease one mechanical load must be } \\
\text { applied to perform a ratchet limit } \\
\text { assessment. }\end{array}$ \\
\hline Dismiss
\end{tabular}

Fig. 3. Main dialog box and possible error messages 

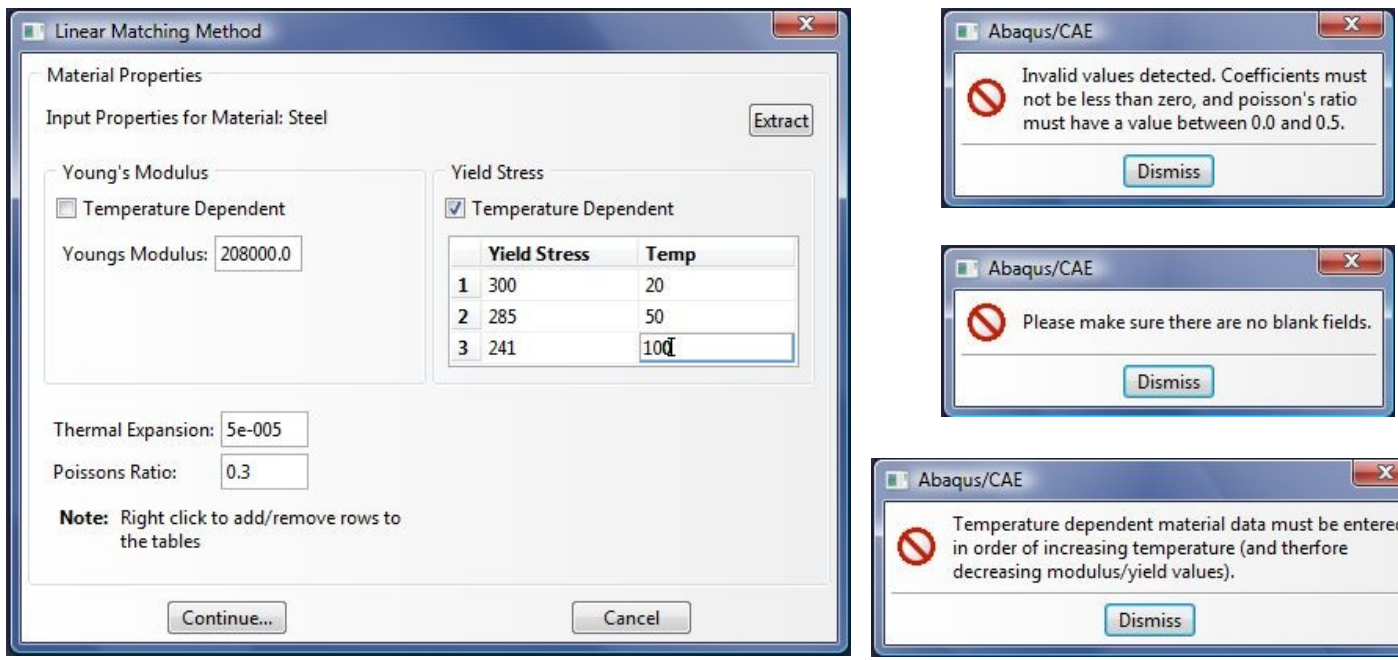

\begin{tabular}{|c|}
\hline Abaqus/CAE \\
$\begin{array}{l}\text { Temperature dependent material data must be entered } \\
\text { in order of increasing temperature (and therfore } \\
\text { decreasing modulus/yield values). }\end{array}$ \\
\hline Dismiss
\end{tabular}

Fig. 4. Material properties dialog box and possible error messages 


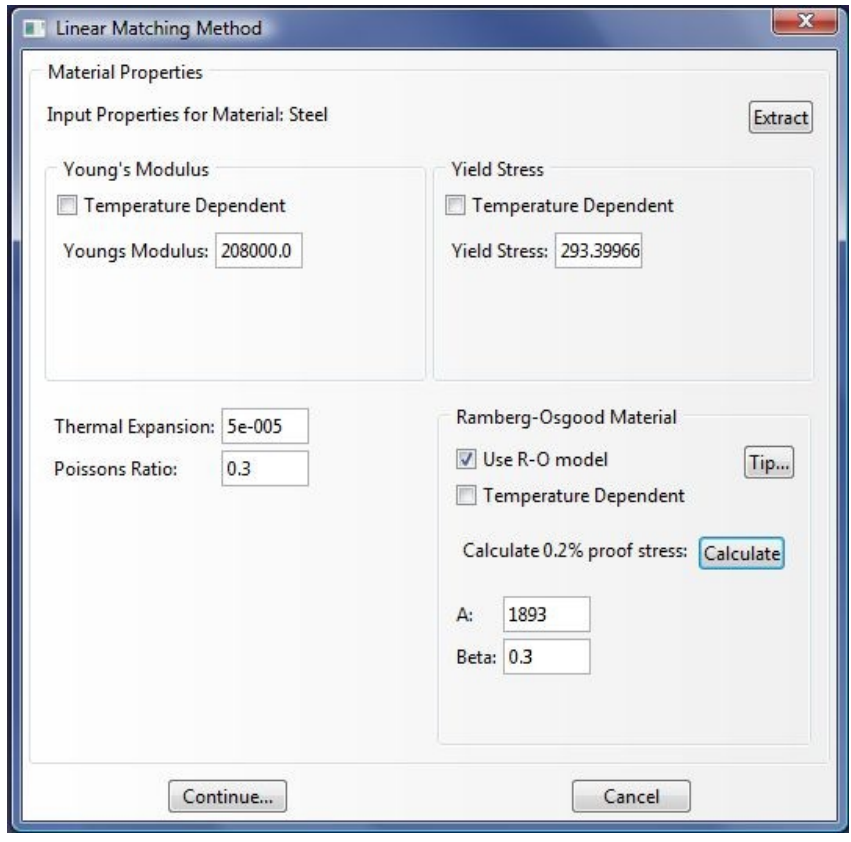

a)

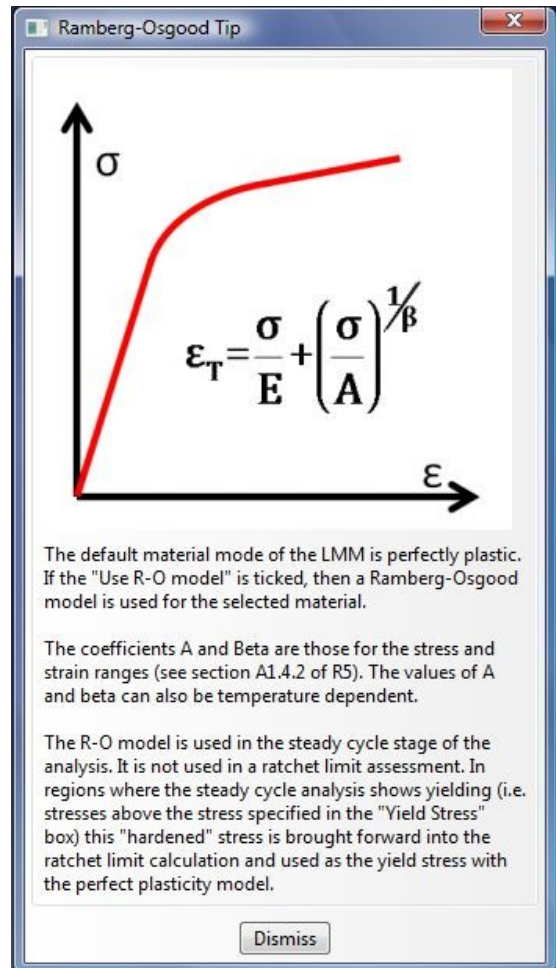

Fig. 5. a) Material properties dialog with Ramberg Osgood option; b) "Tip" box 


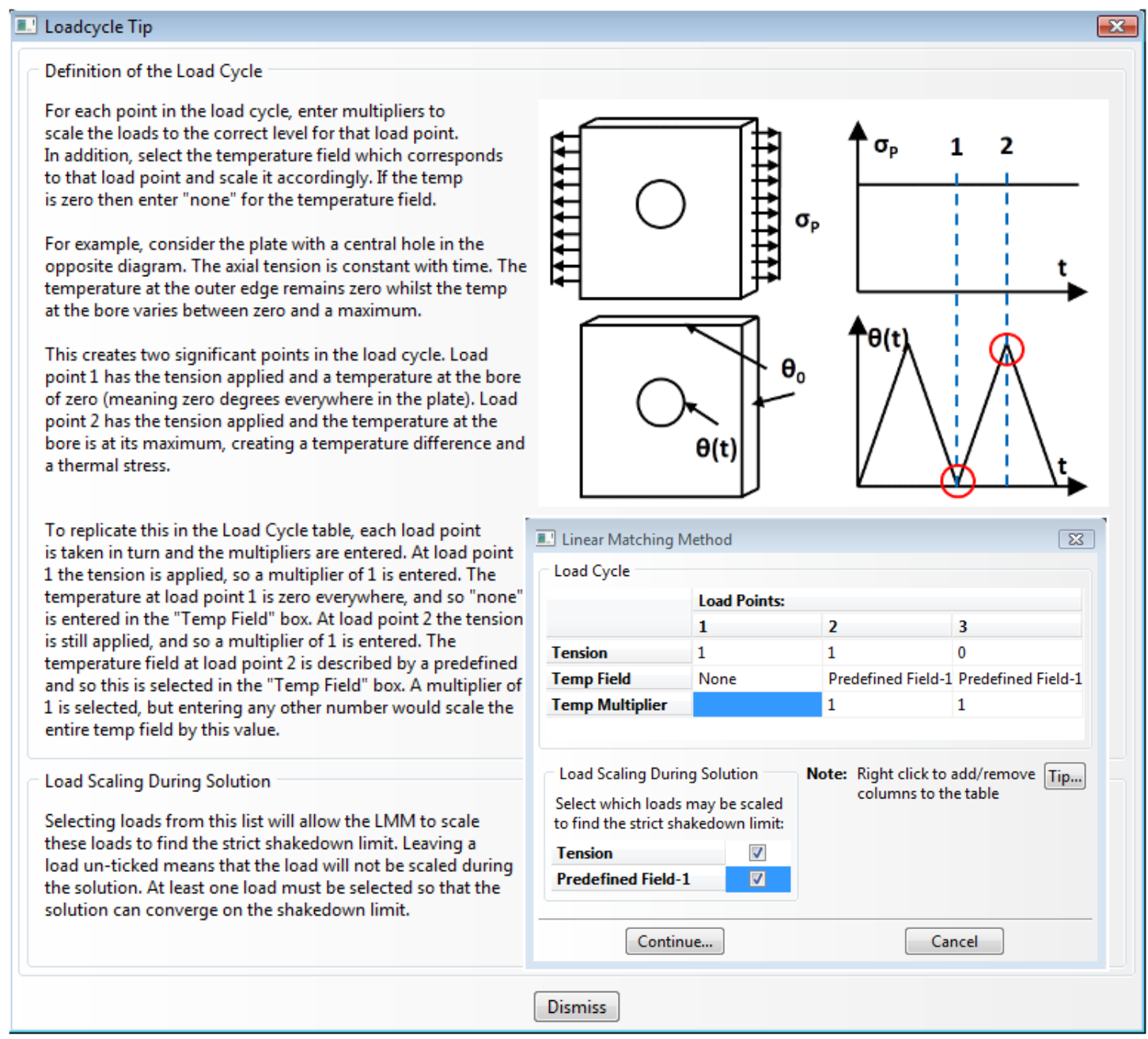

Fig. 6. Loadcycle dialog box and "Tip" box for Loadcycle definition 


\begin{tabular}{|c|c|c|}
\hline \multicolumn{2}{|l|}{ II Linear Matching Method } & $x$ \\
\hline \multicolumn{3}{|l|}{ Analysis Paramters } \\
\hline Job Name: & Sample_Name & \\
\hline Max Number of Increments: & 300 & \\
\hline Select working directory: & Ciljob & Select... \\
\hline \multicolumn{3}{|l|}{ Convergence Level: } \\
\hline \multicolumn{3}{|c|}{ difference between consecutive UB } \\
\hline \multicolumn{3}{|c|}{ \% $\%$ difference between UB and LB } \\
\hline \%Diff: 5 & \multicolumn{2}{|c|}{ Tір... } \\
\hline \multicolumn{3}{|c|}{$\begin{array}{l}\text { Clicking OK converts the model and creates an analysis } \\
\text { job. During solution, please refer to the data tab in the } \\
\text { monitor dialog or the ddat file for load multipliers and } \\
\text { odb outputs. }\end{array}$} \\
\hline $\mathrm{OK}$ & Cance & \\
\hline
\end{tabular}

a)

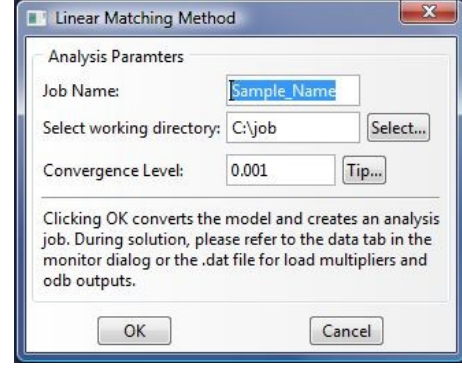

b)

\begin{tabular}{|c|c|}
\hline II Linear Matching Method & $x$ \\
\hline \multicolumn{2}{|l|}{ Analysis Paramters } \\
\hline Job Name: & Sample_Name \\
\hline Max Number of Increments: & 350 \\
\hline Select working directory: & Select... \\
\hline \multicolumn{2}{|c|}{ Steady Cycle Convergence Level: } \\
\hline Steady Cycle Convergence: & Tiр... \\
\hline \multicolumn{2}{|c|}{ Ratchet Limit Convergence Level: } \\
\hline \multicolumn{2}{|c|}{ difference between consecutive UB } \\
\hline \multicolumn{2}{|c|}{ ○ \% difference between UB and LB } \\
\hline \%Diff: 5 & o... \\
\hline \multicolumn{2}{|c|}{$\begin{array}{l}\text { Clicking OK converts the model and creates an analysis } \\
\text { job. During solution, please refer to the data tab in the } \\
\text { monitor dialog or the dat file for load multipliers and } \\
\text { odb outputs. }\end{array}$} \\
\hline OK & Cancel \\
\hline
\end{tabular}

Fig. 7. Analysis parameters dialog boxes for a) strict shakedown; b) steady state cycle; c) steady state cycle + ratchet analysis 


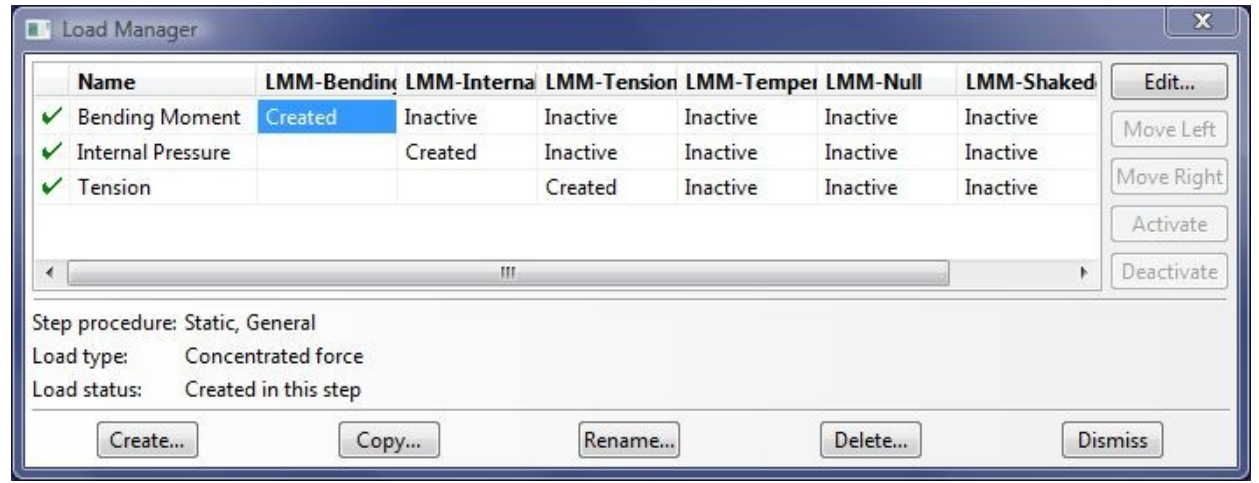

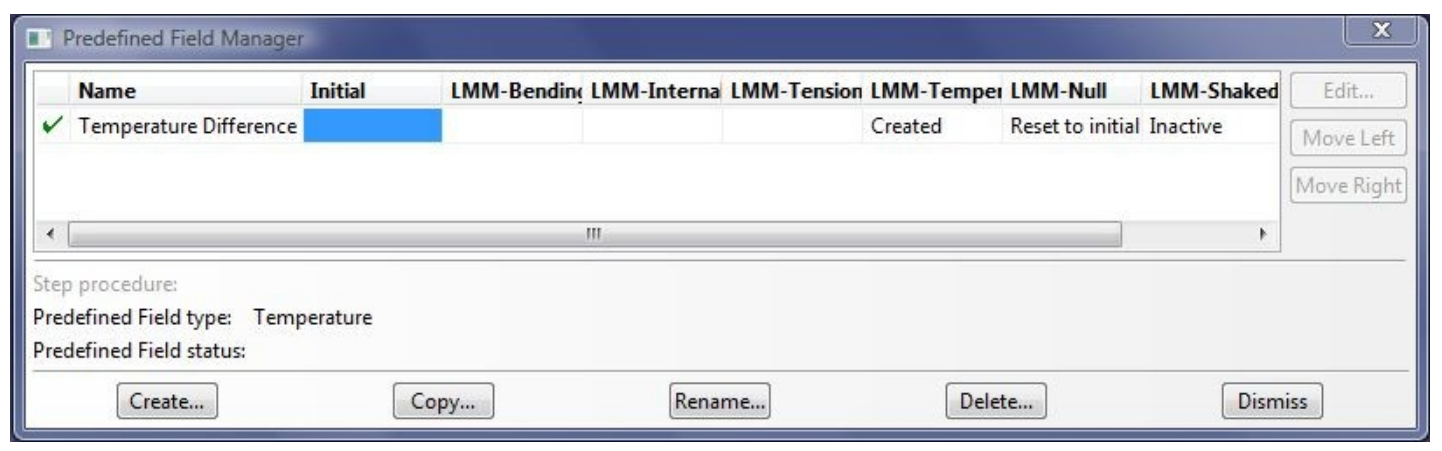

Fig. 8. Load and predefined field manager dialogs 


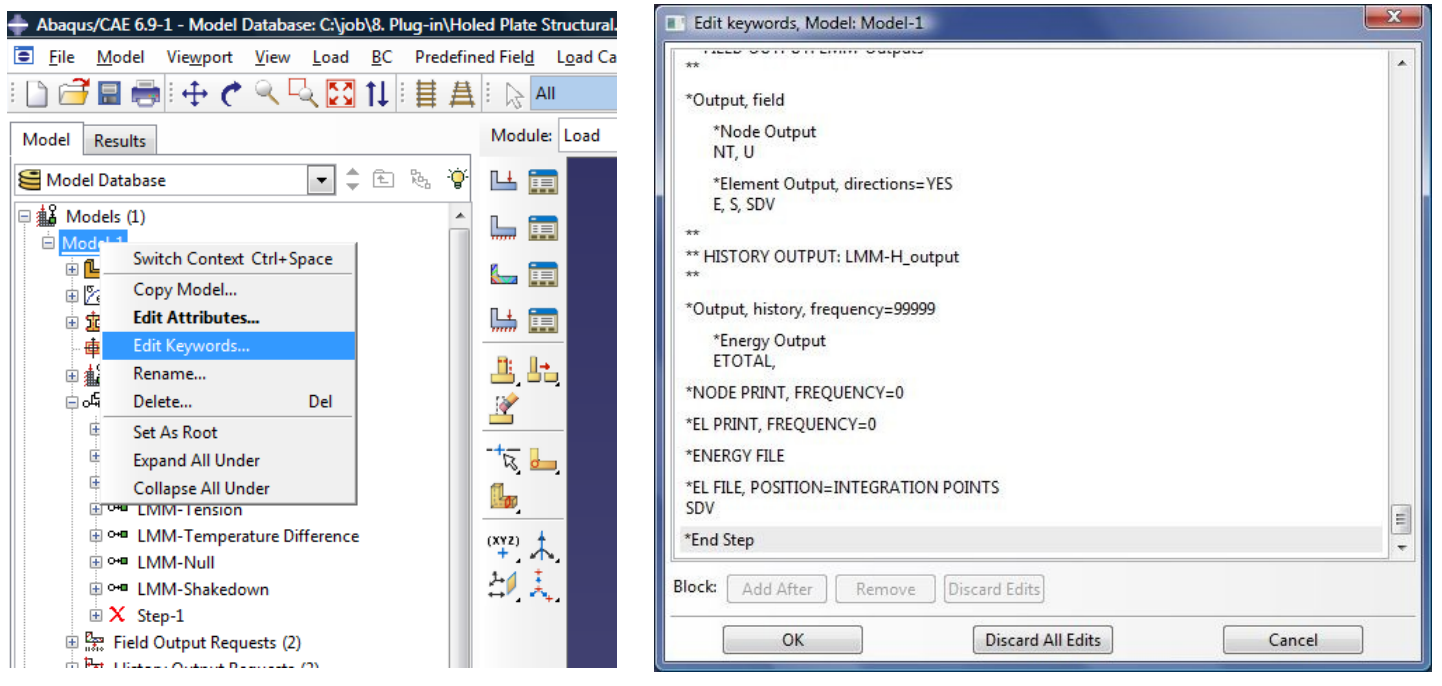

Fig. 9. Keyword block after the *ENERGY FILE command has been added 


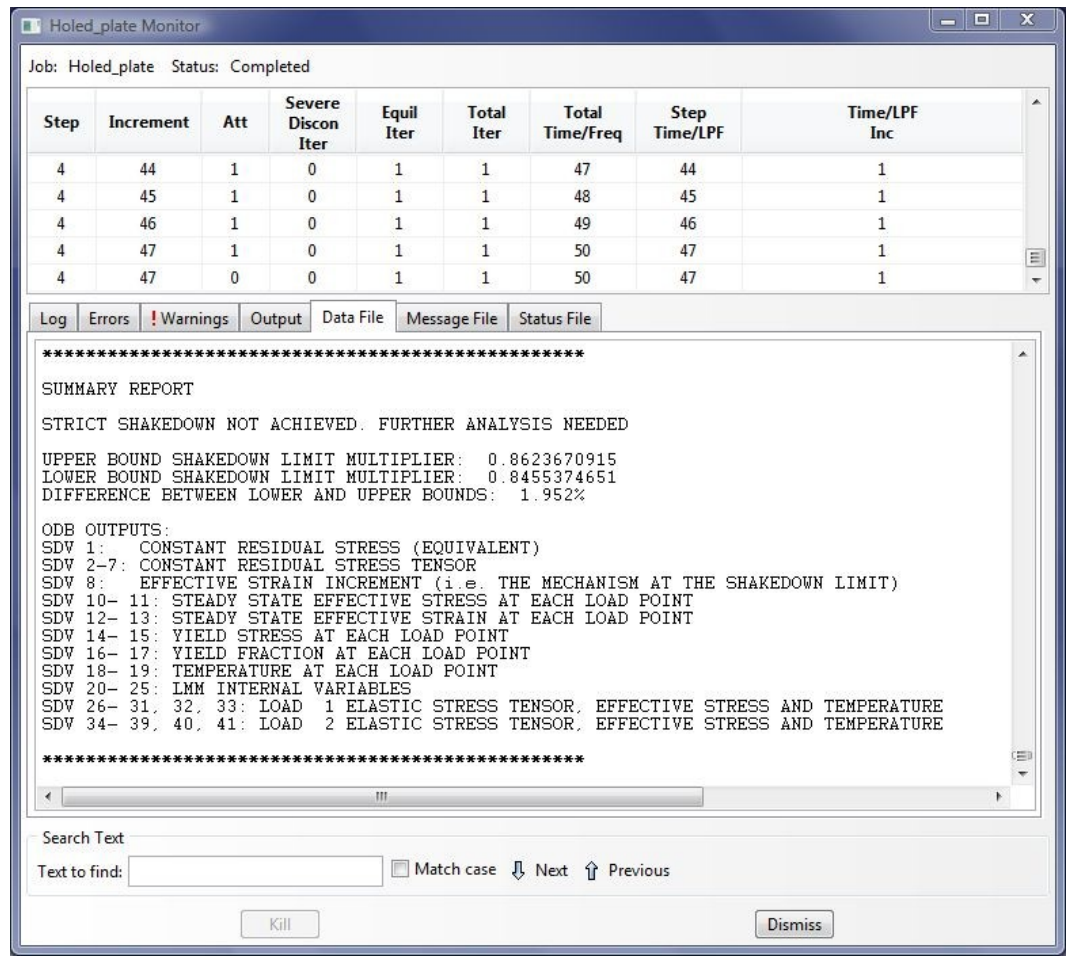

Fig. 10. Monitor dialog box showing the LMM summary report 